\title{
Um Componente para Gerência de Processos de Negócio em Sistemas de Informação
}

\author{
Juliano Lopes de Oliveira ${ }^{1}$, Luiz Fernando Batista Loja ${ }^{1}$, Sofia Larissa da Costa $^{1}$, \\ Valdemar Vicente Graciano Neto ${ }^{1}$
}

\author{
${ }^{1}$ Instituto de Informática - Universidade Federal de Goiás (UFG) \\ Caixa Postal 131 - Campus II - CEP 74001-970 - Goiânia - GO - Brasil \\ Universidade Federal de Goiás - UFG \\ \{juliano,luizloja, sofiacosta, valdemarneto\}@inf.ufg.br
}

\begin{abstract}
Resumo. Construir e manter software para gerenciar Processos de Negócio $(P N)$ em Sistemas de Informação (SI) exige esforço e tempo consideráveis. Este artigo descreve um componente de software que utiliza uma abordagem de engenharia dirigida por modelos para apoiar a gerência de PN no contexto de um framework para desenvolvimento e evolução de SI. O componente Gerenciador de Processos permite a definição de modelos de PN usando uma linguagem de alto nível. Modelos conceituais são integrados com os demais componentes do framework para gerar as aplicações de SI. Este componente apresenta melhorias importantes com relação a outras ferramentas de Gerenciamento de PN, tais como o suporte à modelagem colaborativa e a capacidade de execução de processos empíricos e ad hoc. Além disso, a integração entre o componente Gerenciador de Processos e os demais componentes aumenta significativamente a produtividade no desenvolvimento e manutenção de SI.
\end{abstract}

\begin{abstract}
Building and maintaining software to manage Business Processes $(B P)$ in Information Systems (IS) requires considerable effort and time. This paper describes a software component that uses a Model Driven Engineering (MDE) approach to support business processes management in IS into the context of an IS development and evolution application framework. The Process Manager component allows the definition of BP models using a high-level language. These conceptual models are integrated with other components of the framework to generate the IS applications. The implemented Process Manager component has important improvements comparing to other BP management tools, such as support for collaborative modeling and support for execution of empirical and ad hoc processes. Furthermore, the integration of the Process Manager component and the other framework components significantly increases productivity in IS software development and maintenance.
\end{abstract}

\section{Introdução}

Sistemas de Informação (SI) são construídos para apoiar a troca de informações sobre o negócio de uma organização, provendo o conhecimento necessário para a operação e para a tomada de decisão sobre o negócio. Eles consistem de conjuntos de dados e de processos que coletam, armazenam, transformam e distribuem informações (principalmente) por meio de formulários [Sølvberg 2010]. 
A construção de software para SI envolve, entre outros aspectos, a especificação e a modelagem de dados, funções, regras e processos de negócio, além de mecanismos que disponibilizem estas informações para interação dos usuários com o sistema.

O Grupo de Pesquisa em Engenharia de Software do Instituto de Informática da Universidade Federal de Goiás (INF/UFG) tem pesquisado sobre a construção de componentes para apoiar o desenvolvimento de software para SI contemplando estes aspectos. Neste sentido, um framework para criação de software específico de domínio foi construído para apoiar o desenvolvimento de SI baseado em modelos de alto-nível [Almeida et al. 2009].

Esse framework dá suporte à geração automática e à manutenção de software de SI usando modelos conceituais. Ele gera uma porção considerável do software de SI empregando metamodelos que descrevem conceitualmente aspectos de SI relacionados com os conceitos de negócio, com as regras de negócio e com as interfaces de usuário.

Apesar do sucesso obtido na aplicação desse framework para a construção de SI voltado para o controle de agronegócio, foi identificada uma limitação que dificulta a geração de uma parte significativa do software de SI, que é relacionada à definição e execução de processos de negócio (PN). Para superar esta limitação foi conduzida uma refatoração no framework [Graciano Neto et al. 2010], ocasionando a concepção de dois novos metamodelos [Loja et al. 2010, da Costa et al. 2010] e a implementação de um componente de gerência de PN.

Este artigo descreve os resultados do processo de refatoração que levaram ao desenvolvimento do componente de gerência de PN para o framework. Este componente é um software do tipo Business Process Management System (BPMS), que pode ser utilizado para gerenciar, modelar, e executar PN em SI.

O restante deste documento descreve o componente desenvolvido e está organizado da seguinte forma. A Seção 2 apresenta a motivação para a construção do componente em termos de requisitos de software. A Seção 3 descreve uma visão geral das principais características do componente desenvolvido. A Seção 4 apresenta um experimento empregando o componente desenvolvido. Finalmente, a Seção 5 traz as conclusões e indica direções para trabalhos futuros.

\section{Limitações das Ferramentas Atuais e Requisitos para Gerência de PN}

As ferramentas BPMS permitem modelar processos utilizando, em geral, a notação BPMN (Business Process Modeling Notation) [OMG 2009]. O processo é tipicamente modelado através de um editor gráfico. Uma máquina de execução responsabiliza-se por instanciar e executar os processos modelados, distribuindo as tarefas entre seus participantes e interagindo com eles a fim de concluir a execução do processo.

Das várias ferramentas BPMS disponíveis no mercado Bizagi [Bizagi 2010] e Bonita [Bonita 2010] podem ser citadas como exemplos do estado da prática nesse tipo de software. Uma análise aprofundada desse estado da prática pode ser obtido em [Loja 2011]. Ambas as ferramentas apresentam características típicas de BPMS, mas apresentam algumas limitações importantes para esse tipo de ferramenta.

Por exemplo, Bonita não permite a alteração de processos durante sua execução; não apoia a execução de processos $a d$ hoc e empíricos; não permite a distribuição de 
responsabilidades durante a execução das atividades; e impõe várias restrições para o compartilhamento de processos.

Bizagi também não apoia a execução de processos ad hoc, apesar de permitir sua modelagem. Além disso, ela não permite a alteração do fluxo de um processo durante sua execução, dificultando a execução de processos empíricos. Bizagi ainda não apoia a distribuição de responsabilidades durante a execução de atividades de um processo.

Ambas as ferramentas dificultam a modelagem colaborativa, uma vez que os responsáveis pela definição de processos não conseguem constituir processos a partir (de componentes) de processos já definidos. Assim, não é possível reutilizar definições de processos. Além disso, diversos padrões de processo não são implementados por essas ferramentas, tais como processos ad hoc [Russell et al. 2006b].

Além das ferramentas citadas, muitos trabalhos propõem outras abordagens de gerenciamento de PN com base em BPMN [Awad et al. 2009, Tsai et al. 2007, List and Korherr 2006], mas estes também não implementam todos os padrões de processo, não apoiam a reutilização de elementos do processo e a definição de responsabilidades, e não solucionam problemas conhecidos da notação BPMN, tais como a ambiguidade de estruturas [Nuffel et al. 2009].

Com base nas limitações identificadas nas ferramentas BPMS, os principais requisitos identificados para o gerenciador de PN do framework envolvem: 1) o suporte à modelagem colaborativa, que deve permitir reutilização e compartilhamento de componentes para a modelagem colaborativa de PN; 2) a capacidade de alteração de processos durante a sua execução; 3) a distribuição de responsabilidades, com base nas características dos participantes dos processos; e 4) a capacidade de definição e execução de diferentes tipos de processos.

Modelagem e Interação Colaborativa. Ferramentas BPMS têm o objetivo de auxiliar a modelagem e execução de processos, provendo, adicionalmente, um ambiente colaborativo [Loja and de Oliveira 2010]. Tal ambiente deveria proporcionar uma forma de modelar processos cooperativamente, facilitando o compartilhamento de processos e a reutilização de componentes previamente definidos. Porém, a estrutura dos metamodelos de processo adotados nas ferramentas existentes dificulta o compartilhamento, por exemplo, de artefatos de processo. Em geral, estes artefatos são definidos dentro do contexto de um processo específico. Assim, o compartilhamento é feito apenas em nível de processo, limitando, por exemplo, a reutilização de partes de um processo para definir novos processos.

Capacidade de Alteração de Processos em execução. Como PN são flexíveis e mutáveis, sujeitos a rápidas e constantes mudanças, a possibilidade de alterar um processo durante sua execução é uma característica fundamental para ferramentas de BPMS, embora esta característica não seja disponibilizada nas ferramentas analisadas neste trabalho.

Definição e execução de diferentes tipos de processos. Outra característica importante para o contexto de modelagem de processos é o suporte a tipos específicos de processos: processos empíricos, cuja especificação de tarefas é feita durante a própria execução do processo; processos definidos, cuja definição de tarefas é totalmente realizada antes da execução do processo; e processos ad hoc. 
Para lidar com a incerteza inerente ao desenvolvimento de software, Scrum, por exemplo, utiliza processos empíricos para desenvolver produtos de software. As ferramentas de BPMS analisadas oferecem suporte apenas para processos definidos e são inadequadas para processos empíricos.

Processos ad hoc são formados por grupos de atividades que não possuem relações de sequência, ou seja, a ordem do fluxo de execução das atividades de um processo ad hoc é indefinida [OMG 2009]. Essa indefinição dificulta significativamente a implementação deste tipo de processo, pois não há um padrão predefinido para orientar a execução das atividades contidas no processo.

Para habilitar o tratamento de tais tipos de processos, foram utilizados requisitos fundamentais para representação de $\mathrm{PN}$, chamados de padrões de processo [Russell et al. 2006a, van der Aalst et al. 2003]. Expressar tipos diferentes de processos demanda que tais padrões façam parte do poder de expressão de uma ferramenta BPMS. No entanto, as ferramentas analisadas não implementam todos esses padrões.

Distribuição de tarefas de acordo com perfis. Uma das dificuldades que ocorrem durante a execução de um processo de negócio é a alocação de responsabilidades [de Mello and Rocha 2009]. Na abordagem utilizada pelas ferramentas atuais, apenas um participante pode ser alocado para executar cada tarefa ou, no melhor caso, um grupo de participantes pode ser alocado, mas com o mesmo tipo de envolvimento na tarefa, que é o envolvimento de executor da tarefa. Essa abordagem não permite, por exemplo, representar as cadeias hierárquicas das corporações envolvidas nos PN.

É importante que durante a execução das tarefas definidas no processo, os colaboradores envolvidos possam cooperar. Um fator crítico para o sucesso dessa cooperação é a seleção e alocação de colaboradores com perfis apropriados, de forma a assegurar que as habilidades necessárias para a execução da tarefa estejam disponíveis na equipe alocada. Para tanto, as tarefas devem ser distribuídas de acordo com o perfil (conhecimentos, experiência, e habilidades) de cada participante, ressaltando-se que a participação em uma atividade não se limita à sua execução.

O modelo COBIT (Control Objectives for Information and related Technology) [ITGI 2009], por exemplo, permite a interação entre diversos papéis, com diferentes tipos de envolvimento, na execução das tarefas de um processo. COBIT propõe quatro tipos de perfis de interação: responsável (executor), aprovador, interessado e consultado. Os diferentes tipos de interação definem maneiras diferentes do participante se envolver com uma tarefa, representando de maneira mais fiel a realidade das organizações modernas.

\section{Visão Geral do Gerenciador de PN}

A arquitetura de software do componente gerenciador de PN é composta por três módulos principais: o metamodelo de processos; a máquina de execução de processos; e as interfaces que proporcionam a interação com o usuário. O componente integrado ao framework de desenvolvimento de SI torna esses SI orientados a processos.

O metamodelo de PN permite a reutilização e o compartilhamento dos Objetos de Fluxo dos processos, e apoia a modelagem de processos ad hoc e empíricos [Loja et al. 2010]. A máquina de execução provê suporte para todos os tipos de processos concebidos segundo aquele metamodelo. 
A Figura 1a ilustra a tela inicial da interface do componente. No canto superior da tela constam as funcionalidades relacionadas a modelagem e execução de processos, e a participantes e permissões. Do lado esquerdo é possível visualizar os elementos disponíveis para navegação.

A Figura 1b representa uma ampliação da imagem da barra de ferramentas associada à etapa de modelagem de processos e a Figura 1c representa uma visualização mais detalhada das funcionalidades associadas à execução de processos.

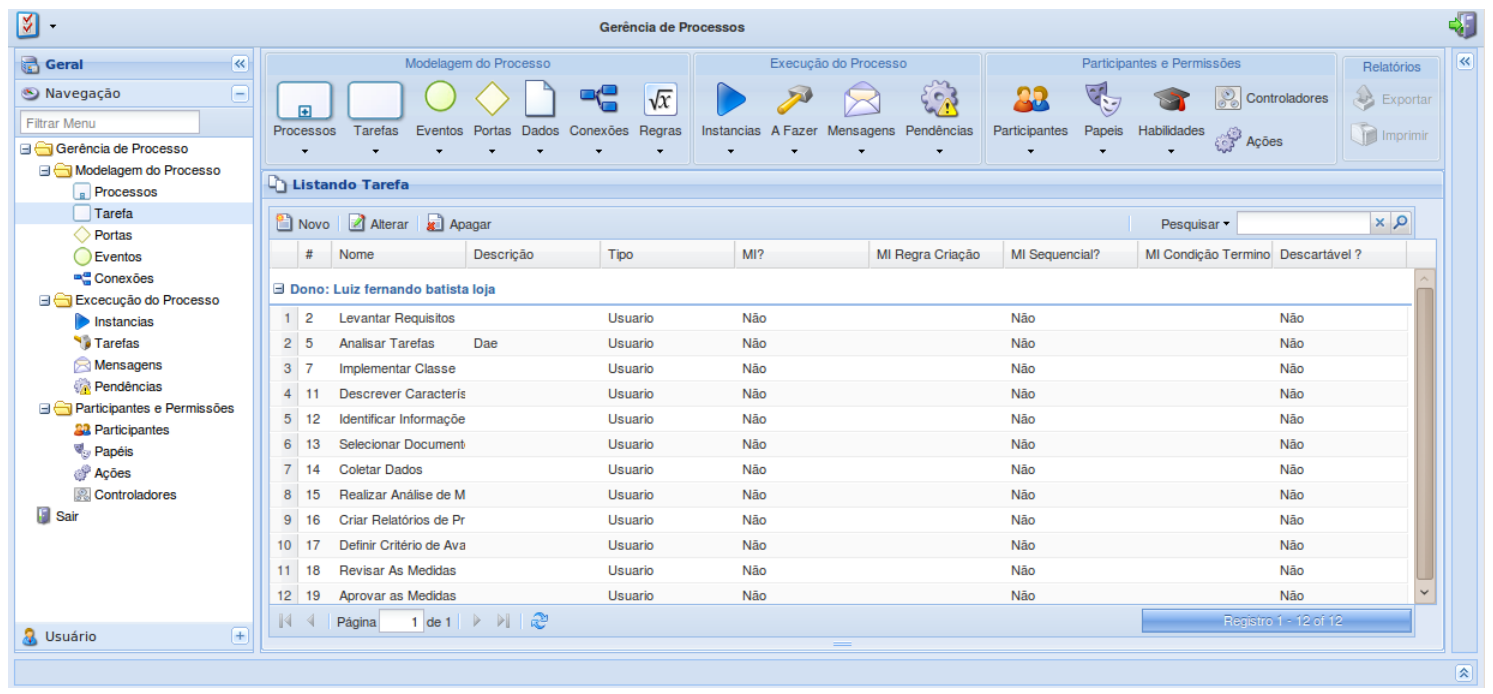

(a) Tela inicial

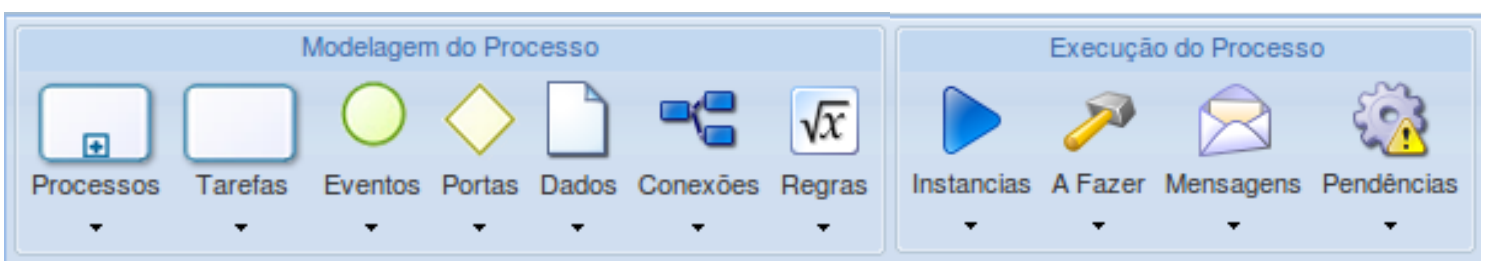

(b) Modelagem

(c) Execução

Figura 1. Tela inicial do componente

A utilização deste componente envolve um processo com três etapas principais: criação, modelagem, e execução de PN.

As funcionalidades relacionadas à criação de processos incluem capacidades para definir processos, tarefas, eventos, portas, objetos de dados e conexões. Para fomentar a reutilização das partes constituintes de um processo na definição de outros processos, a primeira etapa envolve a elaboração das tarefas que farão parte do processo de negócio, bem como a definição de seus participantes, eventos e portas.

Essa reutilização é possível porque, ao contrário da notação BPMN (na qual os elementos do processo são criados em um determinado contexto de processo), os elementos de processo do componente são independentes de contexto. Isso possibilita a reutilização de elementos do processo em outros processos, e o compartilhamento de elementos de processo entre os participantes da execução do processo.

A Figura 2 ilustra a tela de cadastro utilizada para criar um novo processo. Há 
interfaces análogas para o cadastro de eventos, participantes, tarefas e portas.

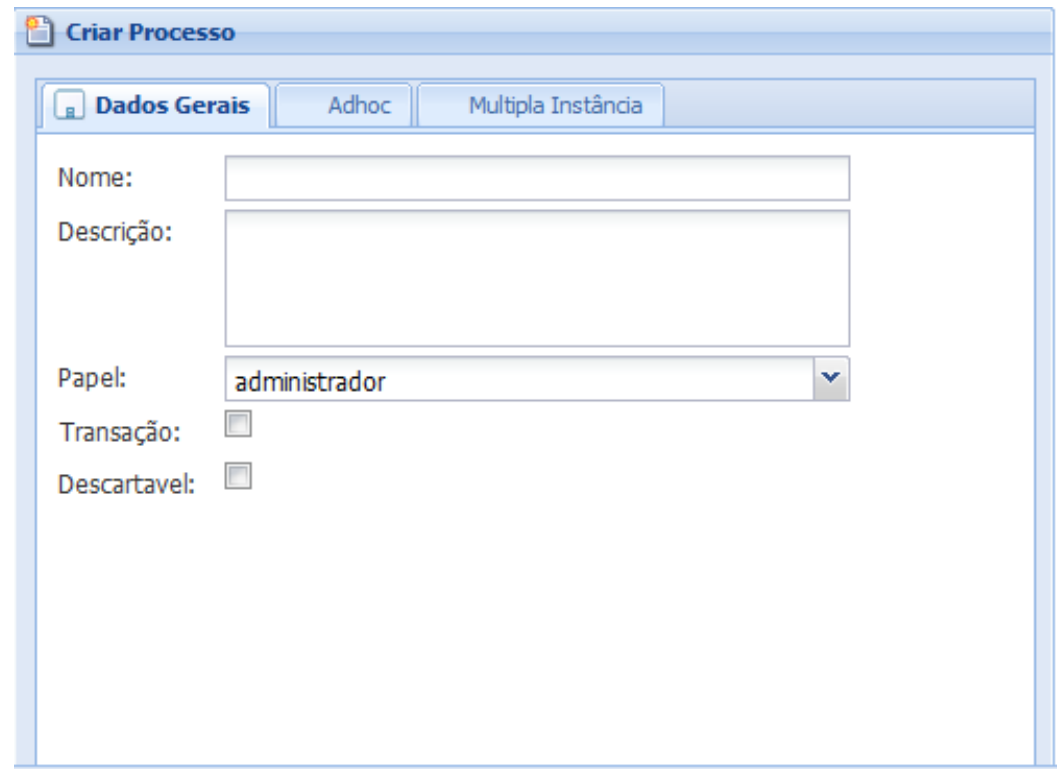

Figura 2. Tela de Cadastro de Processo

O componente adota um subconjunto da notação BPMN para a modelagem de processo [Loja et al. 2010]. Este subconjunto elimina limitações conhecidas de BPMN [Nuffel et al. 2009]. Consequentemente, alguns artefatos de BPMN não estão disponíveis para modelagem, como a construção Text Annotation, que não possui significado equivalente no mundo real, além de baias e raias. No metamodelo proposto, o papel do participante associado à tarefa determina com exatidão quem é o responsável pela tarefa.

Após a etapa de criação do processo e de seus elementos constituintes, inicia-se a etapa de modelagem do processo. Todos os elementos definidos para o processo (na etapa de criação), assim como os elementos pertinentes a processos anteriormente registrados, encontram-se disponíveis para utilização na modelagem. O componente é dotado de um modelador gráfico que permite compor visualmente os elementos de um processo. Assim, é possível montar um processo ligando graficamente os seus elementos.

Os objetos de fluxo formam um repositório de componentes de processo, que podem ser reutilizados para a concepção de novos processos. A Figura 3 ilustra a tela de modelagem gráfica do processo com destaque para o repositório de objetos disponível para utilização do modelador.

Uma vez modelado, o processo pode ser instanciado e executado. A máquina de execução de processos é responsável por instanciar e controlar a execução de qualquer tipo de processo definido de acordo com o metamodelo. Uma característica notável da máquina é a possibilidade de alterar o fluxo do processo durante sua execução. Isso é possível graças à abordagem baseada em tokens [Bendraou et al. 2009] que permite que o processo não seja realmente instanciado na memória da aplicação.

Para executar um processo a máquina de execução lê e executa um passo por vez. Isso significa que toda vez que um token é movido em decorrência de um evento no processo, a máquina de execução analisa o token e define qual o próximo passo na 


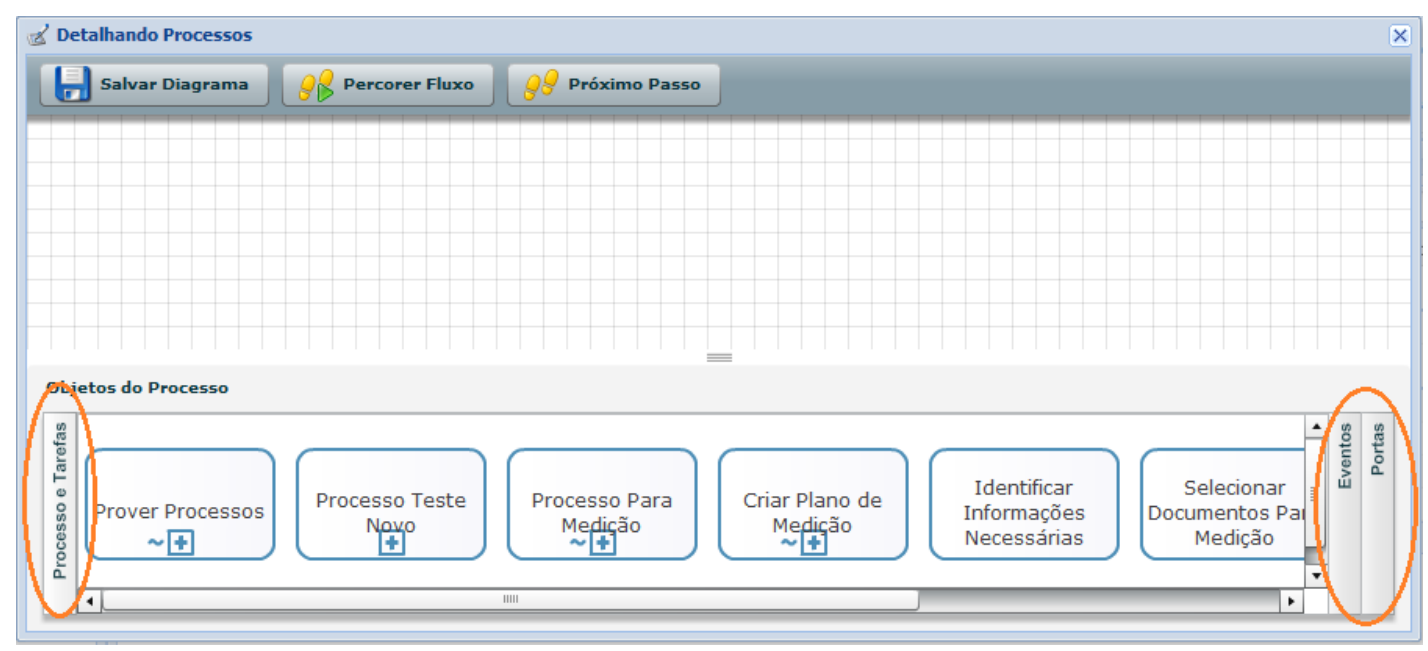

Figura 3. Modelador Gráfico de Processos

execução do processo. Assim, modificações no processo podem ser instantaneamente refletidas em seu fluxo, permitindo a modelagem de processos empíricos.

Os participantes são definidos no momento em que a instância do processo é criada. As responsabilidades das tarefas são distribuídas entre esses participantes durante o fluxo de execução.

\subsection{Potenciais Aplicações do Gerenciador de PN}

Um contexto que pode se beneficiar das funcionalidades disponibilizadas pelo componente gerenciador de PN é o de melhoria de processos de software (MPS). O suporte a processos ad hoc e empíricos é importante, por exemplo, para organizações que estão iniciando projetos de MPS, já que estas costumam trabalhar de modo ad hoc. Já as organizações maduras, que buscam a otimização de processos por meio de experimentações, precisam dispor de processos empíricos para avaliar os custos e benefícios de variações introduzidas em um processo.

O modelador gráfico e o suporte a processo ad hoc são essenciais para apoiar o amadurecimento pelo qual uma organização passa durante as primeiras iniciativas de MPS. A sequência de cenas da Figura 4 ilustra três possíveis momentos da transição de um processo ad hoc para um processo definido: 1) no momento inicial as tarefas são conhecidas (estão disponíveis na parte inferior da tela), mas sua ordem ainda é arbitrária; 2) no momento intermediário são feitas ligações entre as tarefas, de modo a orientar a execução do processo; e 3) no momento final o processo definido especifica claramente como ele deve ser executado.

Outro cenário de aplicação do componente contempla instituições que já possuem seus processos definidos, mas ainda são pouco adotados pelos colaboradores. Neste caso, o componente pode auxiliar por permitir a interação remota (via Web) de colaboradores, além de permitir que um colaborador veja as alterações feitas no processo nos momentos em que ele não estava acompanhando a execução do processo. Isso viabiliza o acesso de todos os colaboradores à versão atual de um dado processo, compartilhando conhecimento organizacional e permitindo maior acompanhamento e aderência à execução desse processo. 


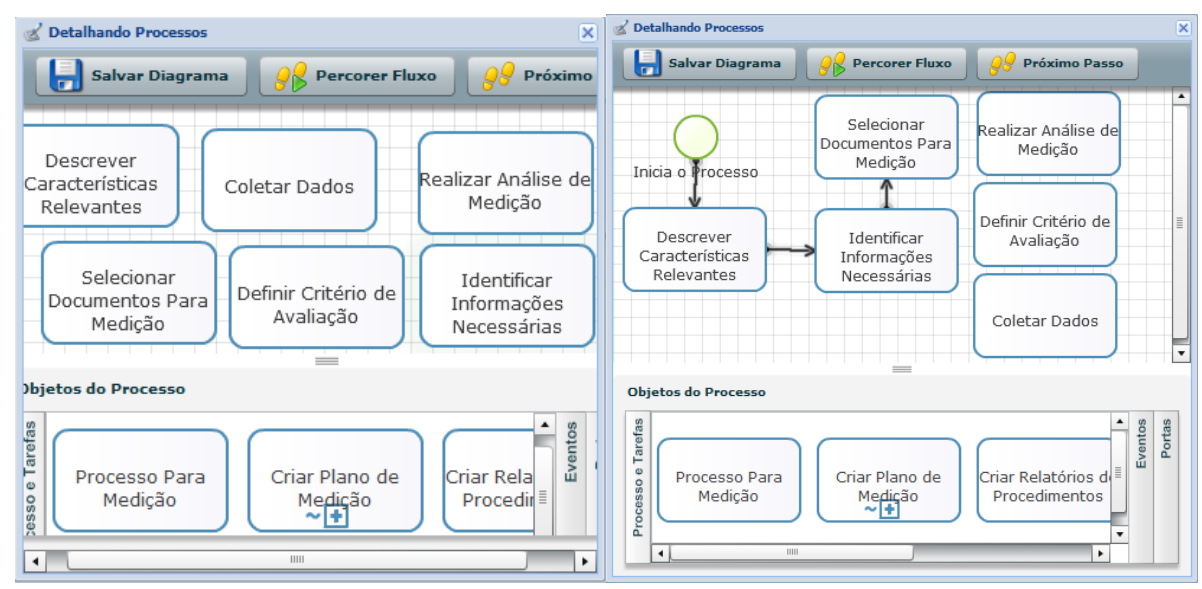

(a) Processo ad hoc

(b) Processo parcialmente definido

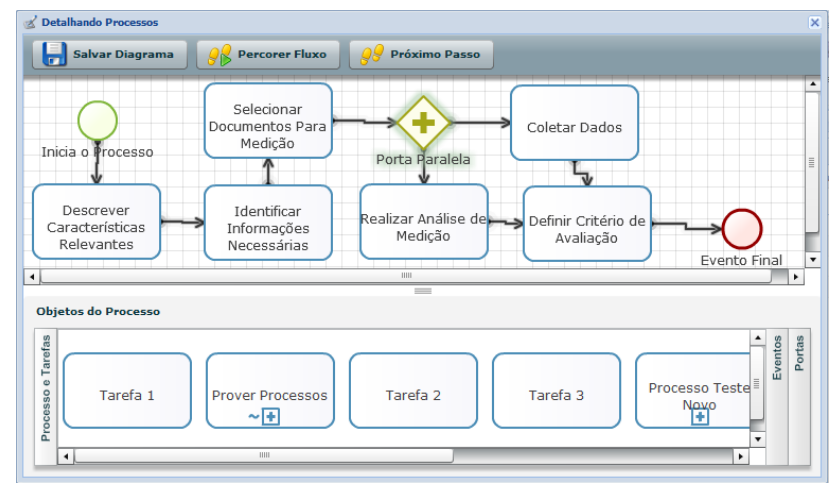

(c) Processo definido

Figura 4. Passos na definição de um processo

A resistência por parte das equipes de projeto para adotar ou modificar um processo de software está relacionada à mudança da cultura organizacional. Mudanças nessa cultura, principalmente nas atividades tradicionalmente executadas de forma ad hoc, são difíceis de serem aceitas pelos envolvidos em iniciativas de MPS. Por isso, na execução de projetos de MPS os servidores mais antigos costumam ser mais resistentes à adoção de novos processos.

O componente descrito pode auxiliar na mudança organizacional por permitir que o gerente de projetos conduza as modificações no processo de forma suave e paulatina. $\mathrm{O}$ apoio à execução de processos ad hoc permite a evolução dos processos da organização, iniciando com um processo mais simples e incrementando-o ao longo do tempo do projeto de MPS.

Os estágios de mudança descritos na Figura 4 amenizam os impactos das mudanças na cultura organizacional, pois permitem estender o tempo de amadurecimento dos processos, possibilitando que colaboradores tenham mais oportunidades para absorver as mudanças, aceitar, implementar e institucionalizar as atividades de um processo, à medida que este evolui e se modifica.

Processos evoluem como um resultado comum da dinâmica organizacional. O componente gerenciador de PN também pode apoiar a evolução dos processos de soft- 
ware. A capacidade que o componente fornece para efetuar modificações em um processo durante a execução de suas instâncias possibilita evoluir a definição de um processo de modo a adaptá-lo à evolução da realidade da organização.

\section{Experimento realizado com o Gerenciador de PN}

Com o objetivo de avaliar a capacidade do componente gerenciador de PN foi conduzido um experimento que visou responder as seguintes questões:

1. O componente cumpre os objetivos propostos (requisitos previstos na Seção 2)?

2. O componente tem poder de expressão suficiente para as necessidades de uma ferramenta BPMS?

Tanto para a avaliação das funcionalidades quanto para a avaliação do poder de expressão da ferramenta, foram definidos procedimentos de coleta de dados e o modo como os dados obtidos deveriam ser analisados. Detalhes sobre esses procedimentos podem ser obtidos em [Loja 2011].

A primeira parte do experimento foi a avaliação de cinco características relacionadas aos diferenciais do componente gerenciador de PN com relação às ferramentas BMPS atuais. Estas características são apresentadas na Tabela 1.

O protocolo do experimento previa inicialmente a modelagem e execução de um processo definido. O processo foi descrito de forma textual para os participantes do experimento e consistia de uma compra de mercadoria envolvendo clientes, atendentes e diferentes formas de pagamento. Durante o experimento, requisitou-se que o processo fosse alterado de diferentes formas, por exemplo, para contemplar uma mudança na forma de pagamento da compra.

Para participar do experimento foram selecionados quatorze usuários que possuíam conhecimentos básicos sobre processo e modelagem de processo. Além disso, todos eles eram profissionais de TI, com formação superior, e experiência de mais de dois anos em desenvolvimento de software e/ou gerência de pessoas e projetos.

Dos participantes do experimento, quatro haviam participado de um projeto de implantação da norma ISO 9001 no setor de atendimento da instituição pública onde o experimento foi aplicado. Os usuários selecionados atuavam em diferentes papéis nessa instituição, incluindo: analistas de sistemas, desenvolvedores, e analistas de negócio.

Para nivelar o conhecimento dos participantes do experimento foi ministrado um curso de três horas de duração sobre gerência e modelagem de processos de negócio, e sobre a utilização do componente de software gerenciador de PN.

Para realizar os procedimentos com todos os participantes foram necessários três dias, com duração média de uma hora por participante. Portanto, foram gastas cerca de quatorze horas na realização dos procedimentos, além das três horas da realização do curso de nivelamento, totalizando dezessete horas de experimento.

Através da análise da Tabela 1 é possível verificar que os participantes do experimento tiveram mais de $80 \%$ de aprovação em relação à presença e correto funcionamento das características avaliadas. Com base nessa análise conclui-se que as funcionalidades propostas foram, de fato, implementadas. As categorias que não totalizaram $100 \%$ refletem o fato dos participantes não terem compreendido a pergunta, ou terem encontrado 


\begin{tabular}{|l|l|}
\hline Característica & Resultado \\
\hline Alterações no modelo do processo durante a execução & $96,42 \%$ \\
\hline Modelagem e Execução de processos empíricos & $100 \%$ \\
\hline Modelagem e Execução de processos ad hoc & $85,71 \%$ \\
\hline Distribuição de responsabilidades aos participantes & $85,71 \%$ \\
\hline Compartilhamento e Reutilização de Elementos do Processo & $92,85 \%$ \\
\hline
\end{tabular}

Tabela 1. Respostas por característica avaliada

uma falha no funcionamento que não permitiu a operação da característica no componente.

Em relação ao poder de expressão, cada padrão de processo modelado foi executado pela máquina de execução do componente e o resultado foi comparado com o comportamento descrito em [van der Aalst et al. 2003]. A implementação de dezenove dos vinte padrões de [van der Aalst et al. 2003] garante que o metamodelo proposto é capaz de expressar uma grande variedade de situações pertinentes ao fluxo de processos.

\section{Conclusões e Trabalhos Futuros}

Este trabalho apresentou um componente de software para gerenciamento de processos de negócio capaz de oferecer um ambiente colaborativo para modelagem e execução de processos no contexto de um framework para síntese de SI utilizando uma abordagem dirigida por modelos. O software apóia a execução de processos empíricos e ad hoc, implementando os principais padrões de processo identificados na literatura [van der Aalst et al. 2003] e superando diversas limitações no tocante ao aspecto de colaboração na modelagem e execução de processos de negócio.

O componente foi desenvolvido para acesso na Web. A implementação foi feita na linguagem Ruby em conjunto com o framework Rails. A interação com o usuário é baseada nos frameworks ExtJs e Flex.

A máquina de execução de PN do componente é capaz de gerenciar a realização de qualquer tipo de processo aderente ao metamodelo de processos definido. Além disso, o mecanismo de execução apoia a interação de mais de um participante por atividade, permitindo até quatro tipos de interação diferentes, com base na definição de responsabilidades proposta no modelo COBIT.

As propostas deste trabalho foram validadas através de um experimento envolvendo quatorze usuários que utilizaram o componente para modelar e executar diversos processos de negócio. Além disso, o componente é capaz de implementar a maioria dos padrões de processo descritos na literatura.

$\mathrm{O}$ framework do qual o componente gerenciador de PN faz parte vem sendo mantido há seis anos pelo Grupo de Pesquisa em Engenharia de Software do INF/UFG. Ele foi refatorado a fim de adicionar características de gerência, definição e execução de processos de negócio, tanto em nível de utilização do framework quanto em nível de geração de código, criando SI orientados a processos.

Melhorias e extensões previstas para as propostas deste trabalho estão relacionadas à máquina de execução, ao componente gerenciador de PN e à experimentação 
do componente em ambientes nos quais processos de negócio são críticos.

Um aprimoramento já previsto para o componente envolve a possibilidade de alterar o modelo do processo individualmente para cada instância. Apesar de a versão atual do componente permitir a alteração dos modelos dos processos enquanto são executados, se um modelo for alterado todas as instâncias que estão utilizando aquele modelo também o serão, o que pode causar efeitos indesejáveis em algumas instâncias.

Além disso, o componente não impõe restrições para alteração dos modelos do processo. Em [Kowalkiewicz et al. 2008] é apresentada uma adaptação de linguagens de expressão de processo com a finalidade de prover suporte a restrição de alteração dos modelos do processo durante a execução. Utilizando essas ideias, seria possível criar um modelo de processo que fosse modificável apenas parcialmente. Por exemplo, a definição de pontos de alteração (ou adaptação) em um processo institucionalizado, contribuiria para a otimização e a extensão deste processo, que poderia ser utilizado como um modelo de referência para processos instanciados a partir dele.

Outra extensão prevista para o componente gerenciador de PN envolve o controle de versão das alterações nos processos, guardando um histórico de alterações, permitindo o acompanhamento da evolução dos processos definidos e gerenciados pelo componente.

\section{Referências}

Almeida, A. C., Boff, G., and Oliveira, J. L. (2009). A Framework for Modeling, Building and Maintaining Enterprise Information Systems Software. In Anais do XXIII Simpósio Brasileiro de Engenharia de Software, pages 115-125. Fortaleza, CE, Brasil.

Awad, A., Grosskopf, A., Meyer, A., and Weske, M. (2009). Enabling Resource Assignment Constraints in BPMN. Technical report, Business Process Technology - Hasso Plattner Institute.

Bendraou, R., Jezéquél, J.-M., and Fleurey, F. (2009). Combining aspect and modeldriven engineering approaches for software process modeling and execution. In Proc. of the International Conference on Software Process: Trustworthy Software Development Processes, ICSP '09, pages 148-160, Berlin, Heidelberg. Springer-Verlag.

Bizagi (2010). Bizagi - business agility. Disponível em: http://www.bizagi.com/. Acesso em: 06 ago. 2010.

Bonita (2010). Bonita. Disponível em: http://www.bonitasoft.org/ . Acesso em: 06 ago. 2010.

da Costa, S. L., Graciano Neto, V. V., Loja, L. F. B., and de Oliveira, J. L. (2010). A Metamodel for Automatic Generation of Enterprise Information Systems. In Anais do I Congresso Brasileiro de Software: Teoria e Prática - I Workshop Brasileiro de Desenvolvimento de Software Dirigido por Modelos, volume 8, pages 45-52, Salvador, BA, Brasil. UFBA.

de Mello, M. S. and Rocha, A. R. (2009). Gestão integrada da melhoria de processos em organizações de software. In Anais do V Workshop Anual do MPS (WAMPS), pages 34 - 41. UNICAMP.

Graciano Neto, V. V., da Costa, S. L., and Oliveira, J. L. (2010). Lições Aprendidas sobre Desenvolvimento Dirigido por Modelos a partir da refatoração de uma ferramenta. In 
Anais do VIII Encontro Anual de Computação - ENACOMP, pages 68-75. Catalão, GO, Brasil.

ITGI (2009). Cobit: Control objective management guidelines maturity model 4.1. http://www.itgi.org. IT Governance Institute.

Kowalkiewicz, M., Lu, R., Baeuerle, S., Kruempelmann, M., and Lippe, S. (2008). Weak dependencies in business process models. pages 177-188.

List, B. and Korherr, B. (2006). An evaluation of conceptual business process modelling languages. In SAC '06: Proceedings of the 2006 ACM symposium on Applied computing, pages 1532-1539, New York, NY, USA. ACM.

Loja, L. F. B. (2011). Sinfonia: uma abordagem colaborativa e flexível para a modelagem e execução de processos de negócio. Master's thesis, Instituto de Informática Universidade Federal de Goiás.

Loja, L. F. B. and de Oliveira, J. L. (2010). Um modelo de colaboração para execução e gerência de processos de negócio em organizações. In Anais do Workshop de Teses e Dissertações em Sistemas Colaborativos no Simpósio Brasileiro de Sistemas Colaborativos (SBSC'10), Belo Horizonte, MG, Brasil.

Loja, L. F. B., Graciano Neto, V. V., da Costa, S. L., and de Oliveira, J. L. (2010). A business process metamodel for Enterprise Information Systems automatic generation. In Anais do I Congresso Brasileiro de Software: Teoria e Prática - I Workshop Brasileiro de Desenvolvimento de Software Dirigido por Modelos, volume 8, pages 37-44, Salvador, BA, Brasil. UFBA.

Nuffel, D. V., Mulder, H., and Kervel, S. V. (2009). Enhancing the formal foundations of bpmn by enterprise ontology. Lecture Notes in Business Information Processing 1865-1348 (Print) - Volume 34 - Advances in Enterprise Engineering III.

OMG (2009). Bpmn information home. Disponível em: http://www.bpmn.org.

Russell, N., Hofstede, A. H. M. T., and Mulyar, N. (2006a). Workflow ControlFlow Patterns: A Revised View. Technical report.

Russell, N., van der Aalst, W. M., ter Hofstede, A. H., and Wohed, P. (2006b). On the Suitability of UML 2.0 Activity Diagrams for Business Process Modelling. In Third Asia-Pacific Conference on Conceptual Modelling (APCCM2006), volume 53 of CRPIT, pages 95-104. ACS.

Sølvberg, A. (2010). On Roles of Models in Information Systems. In Nurcan, S., Salinesi, C., Souveyet, C., and Ralyt, J., editors, Intentional Perspectives on Information Systems Engineering, pages 17 - 38. Springer Publishing Company, Incorporated, 1 edition.

Tsai, C.-H., Luo, H.-J., and Wang, F.-J. (2007). Constructing a BPM environment with BPMN. In Future Trends of Distributed Computing Systems, 2007. FTDCS '07. 11th IEEE International Workshop on, pages 164-172.

van der Aalst, W., ter Hofstede, A., Kiepuszewski, B., and Barros, A. (2003). Workflow patterns. Distributed and Parallel Databases, 14(1):5-51. 